\title{
Finite groups with planar subgroup lattices
}

\author{
Joseph P. Bohanon · Les Reid
}

Received: July 8, 2004 / Revised: June 30, 2005 / Accepted: July 25, 2005

(C) Springer Science + Business Media, Inc. 2006

\begin{abstract}
It is natural to ask when a group has a planar Hasse lattice or more generally when its subgroup graph is planar. In this paper, we completely answer this question for finite groups. We analyze abelian groups, $p$-groups, solvable groups, and nonsolvable groups in turn. We find seven infinite families (four depending on two parameters, one on three, two on four), and three "sporadic" groups. In particular, we show that no nonabelian group whose order has three distinct prime factors can be planar.
\end{abstract}

Keywords Graph · Subgroup graph · Planar · Lattice-planar · Nonabelian group

\section{Introduction}

Dummit and Foote remark that "unlike virtually all groups" $A_{4}$ has a planar Hasse Lattice [6, p. 110]. That is to say, the lattice of subgroups of a given group can rarely be drawn without its edges crossing. Intrigued, the first author began an investigation that led to a Master's thesis written under the supervision of the second author. Recently, others have also considered this problem $[11,14]$. This paper is a revised version of the first author's thesis in which we will completely classify those finite groups having planar lattices.

Before beginning, we need some definitions.

Definition 1.1. The subgroup graph of a group is the graph whose vertices are the subgroups of the group and two vertices, $H_{1}$ and $H_{2}$, are connected by an edge if and only if $H_{1} \leq H_{2}$ and there is no subgroup $K$ such that $H_{1} \supsetneqq K \supsetneqq H_{2}$.

\footnotetext{
J. P. Bohanon $(\bowtie)$

Department of Mathematics, Washington University, St. Louis, Missouri, 63130 e-mail: bohanon@math.wustl.edu

L. Reid $(\square)$

Department of Mathematics, Missouri State University, Springfield, Missouri, 65897

e-mail: les@math.missouristate.edu
} 

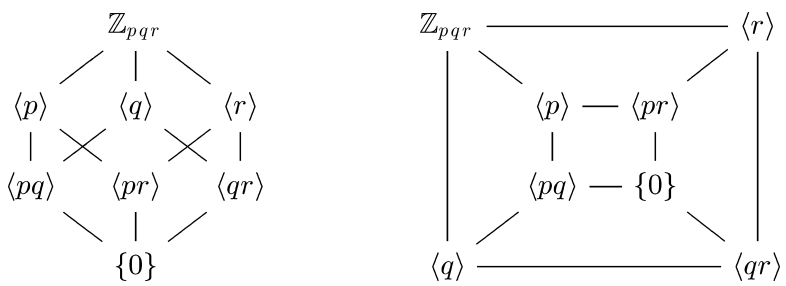

Fig. $1 \mathbb{Z}_{p q r}$ is planar but not lattice-planar

Definition 1.2. We say that a group is planar if its subgroup graph is planar. We say that a group is lattice-planar if its subgroup graph can be drawn in the plane so that all the edges are straight lines, those lines only intersect at vertices, and if $H_{1} \leq H_{2}$, then the $y$-coordinate of $H_{1}$ is less than that of $H_{2}$. (Starr and Turner call this concept "upward planar".)

Note that lattice-planar implies planar.

Example 1.3. Figure 1 shows that $\mathbb{Z}_{p q r}$ is planar, but not lattice-planar.

To show that a group is planar or lattice-planar, we will explicitly exhibit an embedding of its subgroup graph in the plane. To show that a group is not planar, we will use three techniques. Kuratowski's Theorem states that a graph is nonplanar if and only if it contains a subgraph homeomorphic to $K_{5}$ or $K_{3,3}$ [9, p. 103], so if we explicitly exhibit such a subgraph we will have shown nonplanarity. If $G$ has a subgroup that is nonplanar, clearly $G$ must be nonplanar. If we can find an $H \unlhd G$ such that $G / H$ is nonplanar, then $G$ must be nonplanar since it contains a sublattice isomorphic to that of $G / H$.

Example 1.4. Figure 2 shows that $S_{4}$ and $A_{5}$ are nonplanar. We use $S_{X}$ (resp. $A_{X}$ ) to denote the symmetric group (resp. the alternating group) acting on the set $X$. Note that here and in the future we will only include those edges that are part of the subgraph homeomorphic to $K_{3,3}$.

To show that a group is not lattice-planar we will invoke the following theorem of Platt [8].

Theorem 1.5. A finite lattice is lattice-planar if and only if the graph obtained by adding an edge from the minimal element to the maximal element is planar.

Starr and Turner [11] and Bohanon [2] prove the following result for abelian groups.
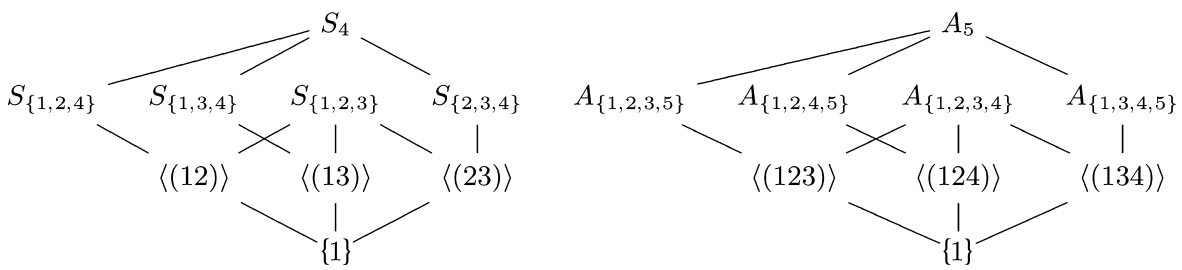

Fig. $2 K_{3,3}$ 's in the lattices of $S_{4}$ and $A_{5}$ 
Theorem 1.6. Up to isomorphism, the only planar abelian groups are the trivial group, $\mathbb{Z}_{p^{\alpha}}$, $\mathbb{Z}_{p^{\alpha} q^{\beta}}, \mathbb{Z}_{p^{\alpha} q r}$, and $\mathbb{Z}_{p^{\alpha}} \times \mathbb{Z}_{p}$, where $p$, $q$, and $r$ denote distinct primes.

The only one of these families that is not lattice-planar is $\mathbb{Z}_{p^{\alpha} q r}$.

The main result of our paper is the following theorem.

Theorem 1.7. Up to isomorphism, the only finite planar groups are the trivial group and

1. $\mathbb{Z}_{p^{\alpha}}, \mathbb{Z}_{p^{\alpha} q^{\beta}}, \mathbb{Z}_{p^{\alpha} q r}, \mathbb{Z}_{p^{\alpha}} \times \mathbb{Z}_{p}$

2. $Q_{8}=\left\langle a, b \mid a^{4}=1, b^{2}=a^{2}, b a b^{-1}=a^{-1}\right\rangle$

3. $Q_{16}=\left\langle a, b \mid a^{8}=1, b^{2}=a^{4}, b a b^{-1}=a^{-1}\right\rangle$

4. $Q D_{16}=\left\langle a, b \mid a^{8}=b^{2}=1, b a b^{-1}=a^{3}\right\rangle$

5. $M_{p^{\alpha}}=\left\langle a, b \mid a^{p^{\alpha-1}}=b^{p}=1, b a b^{-1}=a^{p^{\alpha-2}+1}\right\rangle$

6. $\mathbb{Z}_{q} \rtimes \mathbb{Z}_{p^{\alpha}}=\left\langle a, b \mid a^{q}=b^{p^{\alpha}}=1, b a b^{-1}=a^{i}, \operatorname{ord}_{q}(i)=p\right\rangle$, when $p \mid(q-1)$

7. $\left(\mathbb{Z}_{p} \times \mathbb{Z}_{p}\right) \rtimes \mathbb{Z}_{q}=\langle a, b, c| a^{p}=b^{p}=c^{q}=1, a b=b a, c a c^{-1}=a^{i} b^{j}, c b c^{-1}=a^{k} b^{\ell}$, where $\left(\begin{array}{cc}i & j \\ k & \ell\end{array}\right)$ is an element of order $q$ in $\left.G L_{2}(p)\right\rangle$, when $q \mid(p+1)$,

where $p, q$, and $r$ are distinct primes. The only ones of these that are not lattice-planar are $\mathbb{Z}_{p^{\alpha} q r}$ and $Q D_{16}$.

Our approach will be to first investigate solvable groups. In this case, we will find that we only need consider those groups of order $p^{\alpha}, p^{\alpha} q^{\beta}$, or $p^{\alpha} q^{\beta} r^{\gamma}$, which we investigate in turn. We then consider nonsolvable groups. Using the classification of minimal simple groups we show that there are no nonsolvable planar groups.

\section{Solvable groups}

Recall that a Hall subgroup is a subgroup whose index is relatively prime to its order and that a Sylow basis for a group is a set of Sylow subgroups $\left\{P_{i}\right\}_{i \in \pi(G)}$ (where $\pi(G)$ denotes the set of primes dividing $|G|)$ of $G$ such that $P_{i} P_{j} \leq G$ for all $i, j \in \pi(G)$.

We have the following theorem.

Theorem 2.1. Every solvable group has a Sylow basis $\left\{P_{i}\right\}$. For any $I \subseteq \pi(G), \prod_{i \in I} P_{i}$ is a Hall subgroup of G. Moreover, any two Sylow bases are conjugate.

Proof: [10, p. 229].

Proposition 2.2. There are no solvable planar groups whose orders have more than three distinct prime factors.

Proof: There is a Sylow basis containing four Sylow subgroups $P, Q, R$, and $S$. The sublattice whose vertices are $\{1\}, P, Q, R, S, P Q, P R, P S, Q R, Q S, R S, P Q R, P Q S$, $P R S, Q R S$, and $P Q R S$ is homeomorphic to the lattice of $\mathbb{Z}_{p q r s}$, which is nonplanar by Theorem 1.6.

\section{1. $p$-Groups}

In this section, we will classify the planar and lattice-planar nonabelian groups of order $p^{\alpha}$, where $p$ is a prime. 


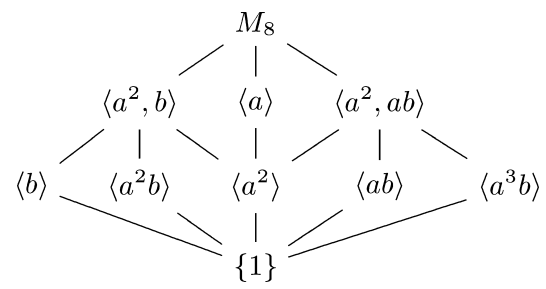

Fig. 3 Lattice of $M_{8}$

Definition 2.3. Let $\alpha$ denote an integer, $\alpha \geq 3$. The quaternionic group of order $2^{\alpha}$ is $Q_{2^{\alpha}}=$ $\left\langle a, b \mid a^{2^{\alpha-1}}=b^{4}=1, a^{2^{\alpha-2}}=b^{2}, b a b^{-1}=a^{-1}\right\rangle$. The quasi-dihedral group of order $2^{\alpha}$ is $Q D_{2^{\alpha}}=\left\langle a, b \mid a^{2^{\alpha-1}}=b^{2}=1, b a b^{-1}=a^{2^{\alpha-2}-1}\right\rangle$. The modular group of order $p^{\alpha}, p$ a prime, is $M_{p^{\alpha}}=\left\langle a, b \mid a^{p^{\alpha-1}}=b^{p}=1, b a b^{-1}=a^{p^{\alpha-2}+1}\right\rangle$.

Lemma 2.4. The modular group $M_{p^{\alpha}}$ is lattice-planar.

Proof: If $p^{\alpha}=8$, then $M_{8} \cong D_{8}$ is lattice-planar by Figure 3 (its subgroup graph is qualitatively different from those of the other modular groups).

It is straightforward to show that Figure 4 gives the subgroup lattice for $M_{p^{\alpha}}, p^{\alpha} \neq 8$. (Note that this lattice is isomorphic to that of $\mathbb{Z}_{p^{\alpha-1}} \times \mathbb{Z}_{p}$.)

It is well known that every $p$-group $G$ contains a central normal subgroup $H$ of order $p$. Since every quotient of a planar group must be planar, it is natural to ask which nonabelian planar $p$-groups $G$ have $G / H$ isomorphic to the planar $p$-groups $\mathbb{Z}_{p^{\alpha-1}} \times \mathbb{Z}_{p}$ or $M_{p^{\alpha}}$. Note that $\mathbb{Z}_{p^{\alpha}}$ cannot occur as such a quotient since $G / H$ being cyclic would force $G$ to be abelian. The following two lemmas are the key to our classification of nonabelian planar and lattice-planar $p$-groups.

Lemma 2.5. If $G$ is nonabelian, $|G|=p^{\alpha}, \alpha \geq 3, H$ is a central subgroup of order $p$, and $G / H \cong \mathbb{Z}_{p^{\alpha-2}} \times \mathbb{Z}_{p}$, then $G$ is planar if and only if $G \cong M_{p^{\alpha}}$ or $Q_{8}$. These groups are all lattice-planar.

Proof: If $\alpha=3$ and $p=2$, the only nonabelian groups are (up to isomorphism) the (latticeplanar) modular group and $Q_{8}$ which is lattice-planar by Figure 5 .

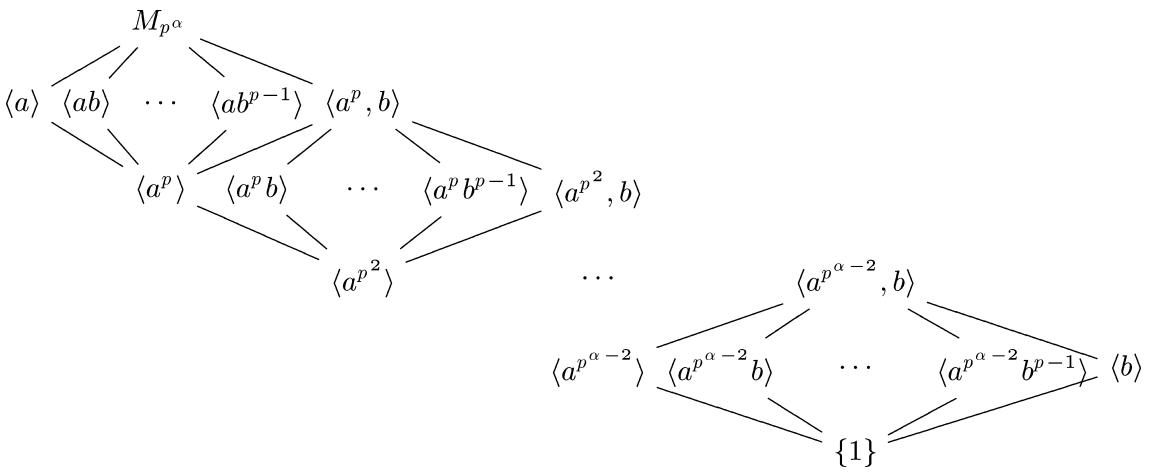

Fig. 4 Lattice of $M_{p^{\alpha}}$ 


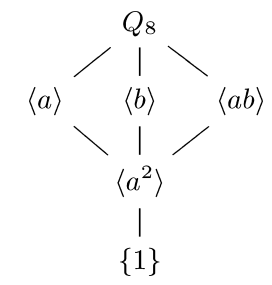

Fig. 5 Lattice of $Q_{8}$

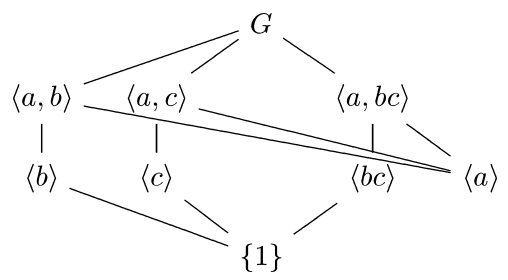

Fig. $6 K_{3,3}$ in the Lattice of $\left(\mathbb{Z}_{p} \times \mathbb{Z}_{p}\right) \rtimes \mathbb{Z}_{p}$

Table 1

\begin{tabular}{ll}
\hline Case & Nonplanar subgroup or subgraph \\
\hline$j=0$ & $\left\langle a^{p}, b, c\right\rangle \cong \mathbb{Z}_{p^{\alpha-3}} \times \mathbb{Z}_{p} \times \mathbb{Z}_{p}$ \\
$j \neq 0, k \neq 0, \alpha>4$ & $\left\langle a^{p}, b\right\rangle \cong \mathbb{Z}_{p^{\alpha-3}} \times \mathbb{Z}_{p^{2}}$ \\
$j \neq 0, k \neq 0, \alpha=4$ & Figure 7 \\
\hline
\end{tabular}

If $\alpha=3$ and $p$ is odd, there are (up to isomorphism) two nonabelian groups of order $p^{3}$. They are the (lattice-planar) modular group and $\left(\mathbb{Z}_{p} \times \mathbb{Z}_{p}\right) \rtimes \mathbb{Z}_{p}=\langle a, b, c| a^{p}=b^{p}=$ $\left.c^{p}=1, b a=a b, c a=a c, c b c^{-1}=a b\right\rangle[3$, p. 145]. Figure 6 shows that the second group is not planar.

Let $H=\langle c\rangle$ with $c^{p}=1$. By our assumption on $G / H$, we may choose $a, b \in G$ such that $a^{p^{\alpha-2}}=c^{i}, b^{p}=c^{j}$ and $b a b^{-1}=a c^{k}$, with $i, j, k \in\{0,1, \ldots, p-1\}$.

If $i \neq 0$, then $a$ has order $p^{\alpha-1}$. According to Burnside, the only nonabelian group of order $p^{\alpha}, p$ odd, with a cyclic subgroup of order $p^{\alpha-1}$ is $M_{p^{\alpha}}$ [3, pp. 134-135]. The only nonabelian groups of order $2^{\alpha}$ that have a cyclic subgroup of order $2^{\alpha-1}$ are $D_{2^{\alpha}}, Q D_{2^{\alpha}}, M_{2^{\alpha}}$ and $Q_{2^{\alpha}}$ [3, p. 135]. But if $G \cong D_{2^{\alpha}}, Q D_{2^{\alpha}}$ or $Q_{2^{\alpha}}, G$ contains a unique central subgroup $H$ of order $p$ and in each case $G / H \cong D_{2^{\alpha-1}}$, which contradicts the fact that $G / H$ is abelian.

Now suppose $i=0$. Note that in all cases $a^{p}$ is central, since $c$ is central and $b a^{p} b^{-1}=$ $\left(b a b^{-1}\right)^{p}=\left(a c^{k}\right)^{p}=a^{p} c^{k p}=a^{p}$. Table 1 gives a list of cases and the corresponding subgroup or subgraph that proves nonplanarity. Note that when $j \neq 0$ and $k=0, G$ is abelian, so we need not consider this case.

The case $j \neq 0, k \neq 0, \alpha=4$ requires a bit of explanation. Taking $\ell \equiv j^{-1} \bmod p$ and $m \equiv(k \ell)^{-1} \bmod p$, and letting $x=b^{\ell}$ and $y=a^{-m}$, we have $c=x^{p}$ and our group is $A=\left\langle x, y \mid x^{p^{2}}=y^{p^{2}}=1, y x y^{-1}=x^{p+1}\right\rangle$. Figure 7 shows a $K_{3,3}$ in the subgroup lattice of this group. The lattices for the case $p=2$ and $p$ an odd prime are slightly different since $(x y)^{2}=y^{2}$ in the first case, but $(x y)^{p}=x^{p} y^{p}$ in the second case. 
Lemma 2.6. If $G$ is nonabelian, $|G|=p^{\alpha}, \alpha \geq 4, H$ is a central subgroup of order $p$, and $G / H \cong M_{p^{\alpha-1}}$, then $G$ is planar if and only if $G \cong Q_{16}$ or $Q D_{16} \cdot Q_{16}$ is lattice-planar, but $Q D_{16}$ is not.

Proof: Analogously to the previous lemma, we have $H=\langle c\rangle$ with $c^{p}=1$ and $a, b \in G$ such that $a^{p^{\alpha-2}}=c^{i}, b^{p}=c^{j}$ and $b a b^{-1}=a^{p^{\alpha-3}+1} c^{k}$, with $i, j, k \in\{0,1, \ldots, p-1\}$. If $i \neq 0$, as in the previous lemma the only possibilities for $G$ are $M_{p^{\alpha}}, D_{2^{\alpha}}, Q_{2^{\alpha}}$, or $Q D_{2^{\alpha}}$. Since $M_{p^{\alpha}} / H$ is abelian, this case cannot occur. For the remaining three types, $G / H \cong D_{2^{\alpha-1}}$ which is not isomorphic to $M_{2^{\alpha-1}}$ unless $\alpha=4$.

We have $D_{16}=\left\langle a, b \mid a^{8}=b^{2}=1, b a b^{-1}=a^{7}\right\rangle$ and Figure 8 gives a sublattice homeomorphic to $K_{3,3}$. Figure 9 shows that $Q_{16}$ is lattice planar. An exercise in Dummit and Foote [6, p. 72] produces a lattice for $Q D_{16}$ that is planar if we reposition the edge from $\left\langle a^{4}\right\rangle$ to $\left\langle a^{4}, a^{2} b\right\rangle$ as shown in Figure 10 . To show that $Q D_{16}$ is not lattice-planar we will
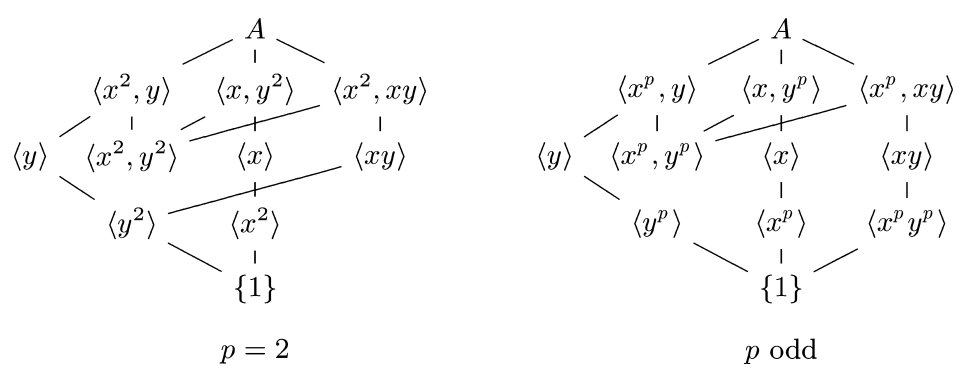

Fig. $7 K_{3,3}$ in the lattice of Group $A$

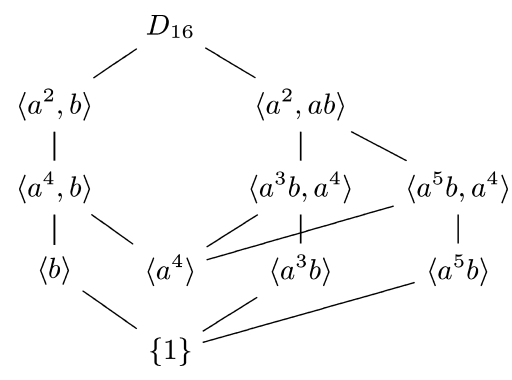

Fig. $8 K_{3,3}$ in the lattice of $D_{16}$

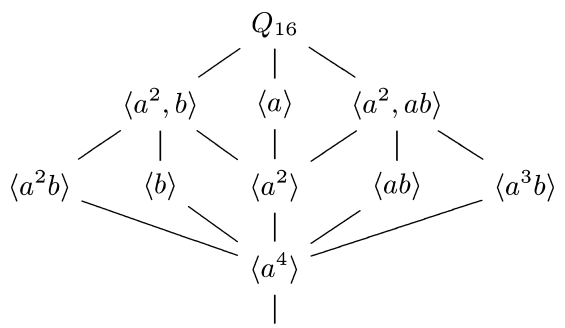

Fig. 9 Lattice of $Q_{16}$ 
invoke Platt's Theorem. We add an additional edge from $\{1\}$ to $Q D_{16}$ and exhibit a subgraph homeomorphic to $K_{3,3}$ in Figure 11.

Now suppose that $i=0$. Note that regardless of the values of $i, j$ and $k, a^{p}$ is central. Table 2 gives a description of the nonplanar subgroup or subgraph in each case.

If $j \neq 0, k=0$ and $\alpha=4, G$ is clearly isomorphic to Group $A$ of Lemma 2.6. If $j \neq$ $0, k \neq 0, \alpha=4$, and $p$ is odd, then taking $\ell \equiv j^{-1} \bmod p, m \equiv(k \ell)^{-1} \bmod p, x=a^{\ell} b^{k \ell^{2}}$, and $y=a^{-m}$ shows (with a bit of calculation) that $G$ is isomorphic to Group $A$. The fact that $\left(a^{s} b^{t}\right)^{p+1}=a^{(p+1) s} b^{(p+1) t}$ is key to this calculation, but this only holds for $p$ odd.

Theorem 2.7. Up to isomorphism, the only nonabelian planar p-groups are are $M_{p^{\alpha}}, Q_{8}$, $Q_{16}$, and $Q D_{16}$. The only one of these that is not lattice-planar is $Q D_{16}$.

Proof: Let $|G|=p^{\alpha}$ and let $H$ be a central subgroup of order $p$, generated by $c$. We will induct on $\alpha$. As noted earlier $G / H$ must be planar and cannot be cyclic (since $G$ is nonabelian).

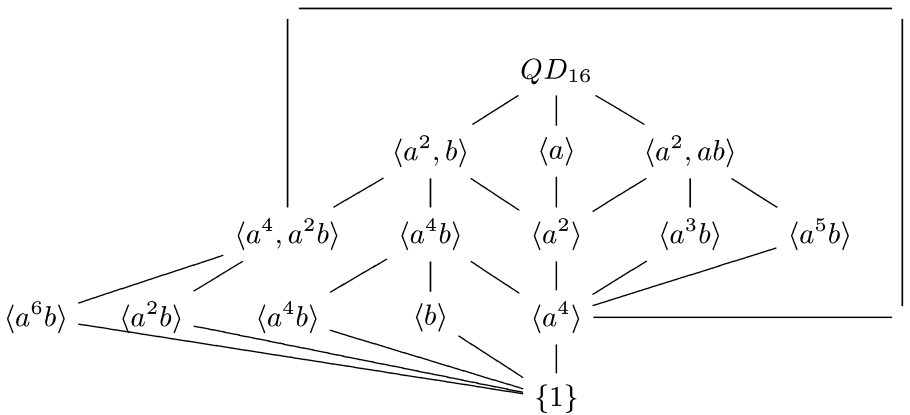

Fig. 10 Re-arranged lattice of $Q D_{16}$

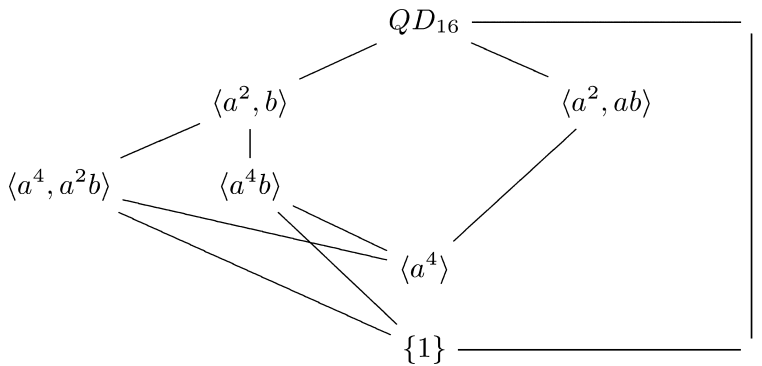

Fig. $11 K_{3,3}$ showing $Q D_{16}$ is not lattice-planar

Table 2

\begin{tabular}{ll}
\hline Case & Nonplanar subgroup or subgraph \\
\hline$j=0$ & $\left\langle a^{p}, b, c\right\rangle \cong \mathbb{Z}_{p^{\alpha-3}} \times \mathbb{Z}_{p} \times \mathbb{Z}_{p}$ \\
$j \neq 0, \alpha>4$ & $\left\langle a^{p}, b\right\rangle \cong \mathbb{Z}_{p^{\alpha-3}} \times \mathbb{Z}_{p^{2}}$ \\
$j \neq 0, k=0, \alpha=4$ & Figure 7 \\
$j \neq 0, k \neq 0, \alpha=4, p=2$ & $\left\langle a^{2}, b^{2}, a b\right\rangle \cong \mathbb{Z}_{2} \times \mathbb{Z}_{2} \times \mathbb{Z}_{2}$ \\
$j \neq 0, k \neq 0, \alpha=4, p$ odd & Figure 7 \\
\hline
\end{tabular}


Table 3

\begin{tabular}{ll}
\hline Case & Choices for $x$ and $y$ \\
\hline$j=0, k=1$ & $x=a b, y=b$ \\
$j=1, k=0$ & $x=a, y=b$ \\
$j=1, k=1$ & $x=b, y=a$ \\
\hline
\end{tabular}

If $\alpha=3$, this means that $G / H \cong \mathbb{Z}_{p} \times \mathbb{Z}_{p}$, hence $G \cong M_{8}$ or $Q_{8}$ by Lemma 2.5. If $\alpha=4$, then $G / H \cong \mathbb{Z}_{p^{2}} \times \mathbb{Z}_{p}, M_{p^{3}}$, or $Q_{8}$. In the first two cases, Lemma 2.5 and Lemma 2.6 force $G \cong M_{p^{4}}, Q_{16}$, or $Q D_{16}$. We claim there is no planar group $G$ such that $G / H \cong Q_{8}$. There are $a, b \in G$ such that $a^{4}=c^{i}, b^{2}=a^{2} c^{j}$, and $b a b^{-1}=a^{-1} c^{k}$, with $i, j, k=0$ or 1 . If $i=1$, then by [3, pp. 134,135] the only candidates for $G$ are $M_{16}, D_{16}, Q_{16}$, or $Q D_{16}$, but as we saw in the proof of Lemma 2.5, $H$ is uniquely determined in each case and $G / H$ is abelian when $G \cong M_{16}$ and dihedral in the other cases. If $i=j=k=0$, then $G /\left\langle a^{2}\right\rangle \cong \mathbb{Z}_{2} \times \mathbb{Z}_{2} \times \mathbb{Z}_{2}$, which is nonplanar. If $i=0$ and at least one of $j$ or $k$ is nonzero, then $G \cong \mathbb{Z}_{4} \rtimes \mathbb{Z}_{4}=$ $\left\langle x, y \mid x^{4}=y^{4}=1, y x y^{-1}=x^{-1}\right\rangle$ (Table 3 gives the appropriate choices for $x$ and $y$ in each case). Figure 12 shows that this group is not planar.

If $\alpha=5$, then the only new candidates for planar groups would have $G / H \cong Q_{16}$ or $Q D_{16}$. Each of these has a subgroup isomorphic to $Q_{8}\left(\left\langle a^{2}, b\right\rangle\right.$ in the first case and $\left\langle a^{2}, a b\right\rangle$ in the second), so $G$ would contain a subgroup $K$ such that $K / H \cong Q_{8}$, but such a $K$ must be nonplanar by our arguments when $\alpha=4$.

Finally, if $\alpha>5$, then $G / H \cong \mathbb{Z}_{p^{\alpha-2}} \times \mathbb{Z}_{p}$ or $M_{p^{\alpha-1}}$ by induction and $G \cong \mathbb{Z}_{p^{\alpha-1}} \times \mathbb{Z}_{p}$ or $M_{p^{\alpha}}$ by Lemmas 2.5 and 2.6.

\subsection{Groups of order $p^{\alpha} q^{\beta}$}

We need some standard notation and a definition.

Notation 2.8. We will denote the order of an element $x \in \mathbb{Z}_{n}$ by $\operatorname{ord}_{n}(x)$. We will let $n_{p}(G)$ denote the number of Sylow $p$-subgroups in $G$; if there is no possibility of ambiguity we will simply write $n_{p}$.

Definition 2.9. Given a group $G$ and $H_{1}, H_{2}, H_{3}, K \leq G$, we say that $K$ is trivalent with respect to $H_{1}, H_{2}$ and $H_{3}$ if in the subgroup graph of $G$ there are chains of edges from $K$ to each of the $H_{i}$ such that the chains only intersect at $K$.

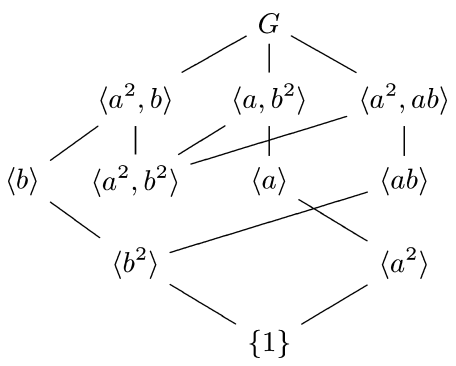

Fig. $12 K_{3,3}$ in the lattice of $\mathbb{Z}_{4} \rtimes \mathbb{Z}_{4}$ 
Before we look at any specific examples, we state two lemmas and a proposition regarding groups of order $p^{\alpha} q^{\beta}$.

Lemma 2.10. If $G$ is a group and $H_{1}, H_{2}, H_{3} \leq G$ such that $H_{i} \not \leq H_{j}$ for $i \neq j$, there is a subgroup of $H_{1}$ and a supergroup of $H_{1}$ that are each trivalent with respect to the $H_{i}$.

Proof: We will denote the subgroup we seek by $D$. If $H_{1} \cap H_{2} \nsubseteq H_{1} \cap H_{3}$, let $D=H_{1} \cap H_{2}$ and our chains are $D \rightarrow H_{1}, D \rightarrow H_{2}, D \rightarrow H_{1} \cap H_{2} \cap H_{3} \rightarrow H_{3}$. If $H_{1} \cap H_{2} \subseteq H_{1} \cap H_{3}$, let $D=H_{1} \cap H_{3}$ and our chains are $D \rightarrow H_{1} \cap H_{2} \cap H_{3} \rightarrow H_{1}, D \rightarrow H_{2}$, and $D \rightarrow H_{3}$. Repeating the proof with $\langle X, Y\rangle$ replacing $X \cap Y$ and reversing the inclusions produces the supergroup.

Proposition 2.11. Let $G$ be a group of order $p^{\alpha} q^{\beta}, \alpha \geq 2$. If $n_{p}>1, G$ has a normal subgroup $K$ of index $p$, and the intersections of three of the Sylow p-subgroups of $G$ with $K$ are distinct, then $G$ is nonplanar.

Proof: Let our three Sylow $p$-subgroups be $P_{1}, P_{2}$ and $P_{3}$. By Lemma 2.10 we can find a subgroup of $P_{1} \cap K$ that is trivalent with respect to $P_{1} \cap K, P_{2} \cap K$, and $P_{3} \cap K$ (since groups of this form have the same order and are distinct, by our hypothesis, we cannot have any containment relationships). By Lemma 2.10, we can find a supergroup of $P_{1}$ that is trivalent with respect to $P_{1}, P_{2}$ and $P_{3}$ (and consequently with respect to $P_{1} \cap K, P_{2} \cap K$ and $P_{3} \cap K$ ). Moreover $P_{1} \cap K, P_{2} \cap K$ and $P_{3} \cap K$ also connect to $K$ (or equivalently, to a subgroup of $K$ ). Note that our common supergroup cannot be $K$ because $K$ has order $p^{\alpha-1} q^{\beta}$ and $P_{i}$ has order $p^{\alpha}$. This provides a homeomorphic copy of $K_{3,3}$ and completes the proof.

We will begin our investigation with groups of order $p^{\alpha} q$, then $p^{2} q^{2}$, and finally $p^{\alpha} q^{\beta}$ with $\alpha, \beta \geq 2$.

\subsubsection{Groups of order $p^{\alpha} q$}

Lemma 2.12. The semi-direct product $\mathbb{Z}_{q} \rtimes_{t} \mathbb{Z}_{p^{\alpha}}=\langle a, b| a^{q}=b^{p^{\alpha}}=1, b a b^{-1}=a^{i}$, $\left.\operatorname{ord}_{q}(i)=p^{t}\right\rangle$, where $p^{t} \mid(q-1)$, is lattice-planar if $t=0$ or 1 and nonplanar if $t>1$.

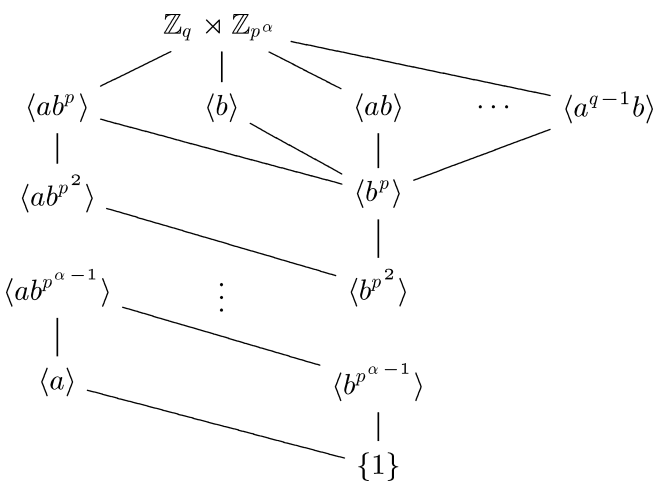

Fig. 13 Lattice of $\mathbb{Z}_{q} \rtimes \mathbb{Z}_{p^{\alpha}}$ 
Every semi-direct product $\mathbb{Z}_{q} \rtimes \mathbb{Z}_{p^{\alpha}}$ is of one of these types. Note that in the future, when $t=1$ we will suppress the subscript.

Proof: When $t=0$, we have the direct product which is planar by Theorem 1.6. It is straightforward to show that Figure 13 gives the subgroup lattice when $t=1$.

When $t>1$, applying Proposition 2.11 with $P_{1}=\langle b\rangle, P_{2}=\langle a b\rangle, P_{3}=\left\langle a^{2} b\right\rangle$, and $K=$ $\left\langle a, b^{p}\right\rangle \unlhd G$ shows that we have a nonplanar group in this case.

Proposition 2.13. Up to isomorphism, the only nonabelian planar groups of order $p^{2} q$ are the semi-direct product $\mathbb{Z}_{q} \rtimes \mathbb{Z}_{p^{2}}$ described in Lemma 2.12 (when $p \mid(q-1)$ ) and

$$
\left(\mathbb{Z}_{p} \times \mathbb{Z}_{p}\right) \rtimes \mathbb{Z}_{q}=\left\langle a, b, c \mid a^{p}=b^{p}=c^{q}=1, a b=b a, c a c^{-1}=a^{i} b^{j}, c b c^{-1}=a^{k} b^{\ell}\right\rangle
$$

where $\left(\begin{array}{cc}i & j \\ k & \ell\end{array}\right)$ is an element of order $q$ in $G L_{2}(p)$ (when $\left.q \mid(p+1)\right)$.

All of these groups are lattice-planar.

Proof: Burnside provides a classification of groups of order $p^{2} q$ [3, pp. 76-80]. First we deal with the case when $p<q$.

Case 1a, $p \nmid(q-1)$ : Sylow's Theorem shows that there are no nonabelian groups in this case.

Case $1 \mathrm{~b}, p \mid(q-1)$, but $p^{2} \nmid(q-1)$ : In this case we have two nonabelian groups. The first is $\mathbb{Z}_{q} \rtimes \mathbb{Z}_{p^{2}}$ which is lattice-planar by Lemma 2.12. We refer to this group as Group 1 of order $p^{2} q$.

The second group in this case is $\langle a, b, c| a^{q}=b^{p}=c^{p}, b a b^{-1}=a^{i}, c a=a c, c b=$ $\left.b c, \operatorname{ord}_{q}(i)=p\right\rangle$. We refer to this group as Group 2 of order $p^{2} q$. The conditions of Proposition 2.11 are satisfied with $P_{1}=\langle b, c\rangle, P_{2}=\langle a b, c\rangle, P_{3}=\left\langle a^{2} b, c\right\rangle$ and $K=\langle a, b\rangle \unlhd G$, so it is nonplanar.

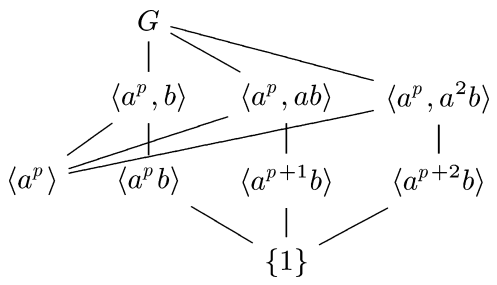

Fig. $14 K_{3,3}$ in the lattice of Group 4 of order $p^{2} q$

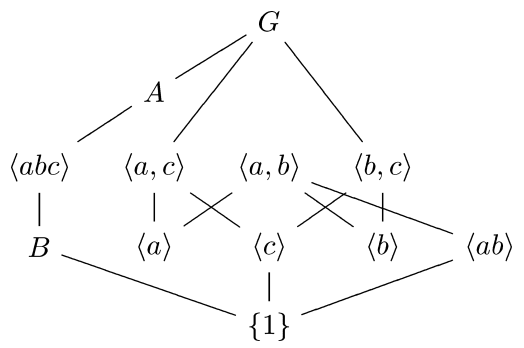

Fig. $15 K_{3,3}$ in the lattices of Groups 5(t) of order $p^{2} q$ 
Case 1c, $p^{2} \mid(q-1)$ : We automatically get both groups from Case $1 \mathrm{~b}$. We also get $\mathbb{Z}_{q} \rtimes_{2} \mathbb{Z}_{p^{\alpha}}$, which is nonplanar by Proposition 2.12. We refer to this group as Group 3 of order $p^{2} q$.

Now consider when $p>q$. We cannot apply Proposition 2.11 , since there is a unique Sylow $p$-subgroup in this case.

Case $2 \mathrm{a}, q \nmid\left(p^{2}-1\right)$ : In this case there are no nonabelian groups.

Case $2 \mathrm{~b}, q \mid(p-1)$ : First, we get $\left\langle a, b \mid a^{p^{2}}=b^{q}=1, b a b^{-1}=a^{i}, \operatorname{ord}_{p^{2}}(i)=q\right\rangle$. We refer to this group as Group 4 of order $p^{2} q$. This is nonplanar by Figure 14 .

Next we have $\langle a, b, c| a^{p}=b^{p}=c^{q}=1, c a c^{-1}=a^{i}, c b c^{-1}=b^{i^{t}}, a b=b a, \operatorname{ord}_{p}(i)=$ $q\rangle$. There are $(q+3) / 2$ isomorphism types in this family (one for $t=0$ and one for each pair $\left\{x, x^{-1}\right\}$ in $\left.\mathbb{F}_{p}^{\times}\right)$. We will refer to all of these groups as Group $5(\mathrm{t})$ of order $p^{2} q$. Figure 15 shows a subgraph homeomorphic to $K_{3,3}$. When $t \neq 0$ or 1 , neither $A$ nor $B$ are present. When $t=0, A$ is absent and $B=\langle a c\rangle$. When $t=1, A=\langle a b, c\rangle$ and $B$ is absent.

Case $2 \mathrm{c}, q \mid(p+1)$ : The only nonabelian group here is $\langle a, b, c| a^{p}=b^{p}=c^{q}=1$, $\left.b a=a b, c a c^{-1}=a^{i} b^{j}, c b c^{-1}=a^{k} b^{\ell}\right\rangle$ where $\left(\begin{array}{cc}i & j \\ k & \ell\end{array}\right)$ has order $q$ in $G L_{2}(p)$. We refer to this group as Group 6 of order $p^{2} q$. This group is lattice-planar by Figure 16.

It should be noted that when $(p, q)=(2,3)$, cases 1 and 2 are not mutually exclusive. There are three nonabelian groups of order 12 up to isomorphism: $T=\mathbb{Z}_{3} \rtimes \mathbb{Z}_{4}$ (Group 1 , lattice-planar), $D_{12}$ (Group 2, nonplanar), and $A_{4}$ (Group 6, lattice-planar).

Proposition 2.14. The only planar groups of the form $\mathbb{Z}_{p^{\alpha}} \rtimes \mathbb{Z}_{q^{\beta}}, \alpha>1, \beta>0$, are the cyclic groups.

Proof: Let $G$ be a planar group of the form $\mathbb{Z}_{p^{\alpha}} \rtimes \mathbb{Z}_{q^{\beta}}$. We have a presentation for $G$ of the form $G=\left\langle a, b \mid a^{p^{\alpha}}=b^{q^{\beta}}=1, b a b^{-1}=a^{i}\right\rangle$, where $i^{q^{\beta}} \equiv 1 \bmod p^{\alpha}$. We will induct on $\alpha+\beta$ beginning with $\alpha+\beta=3$, where the result follows by Proposition 2.13. Suppose that $\alpha+\beta>3$. We must have $\alpha>2$ or $\beta>1$. If $\alpha>2$, then $\left\langle a^{p}, b\right\rangle \cong \mathbb{Z}_{p^{\alpha-1}} \rtimes \mathbb{Z}_{q^{\beta}}$ with $\alpha-1>1$. By induction this subgroup must be cyclic, so $a^{p}=b a^{p} b^{-1}=a^{i p}$ which implies that $i \equiv 1 \bmod p^{\alpha-1}$, i.e. $i=1+k p^{\alpha-1}$. Now $\left\langle a, b^{q}\right\rangle$ is also cyclic (by induction if $\beta>1$, by inspection if $\beta=1$ ). Therefore $a=b^{q} a b^{-q}=a^{i^{q}}$, so $i^{q} \equiv 1 \bmod p^{\alpha}$. But this yields $(1+$ $\left.k p^{\alpha-1}\right)^{q} \equiv 1+q k p^{\alpha-1} \equiv 1 \bmod p^{\alpha}$ which forces $p \mid k$ and $i \equiv 1 \bmod p^{\alpha}$ and our original group is abelian. If $\beta>1$, then $\left\langle a, b^{q^{\beta-1}}\right\rangle$ is cyclic by induction (we need $\beta>1$ for this to be a proper subgroup). The subgroup $H=\left\langle b^{q^{\beta-1}}\right\rangle$ is normal and $G / H \cong\langle a, b| a^{p^{\alpha}}=b^{q^{\beta-1}}=$ $\left.1, b a b^{-1}=a^{i}\right\rangle$. But this group must also be cyclic by induction, hence $i=1$ and therefore $G$ is cyclic as well.

Proposition 2.15. Up to isomorphism, the only planar groups of order $p^{\alpha} q$ with $\alpha \geq 3$ are $\mathbb{Z}_{p^{\alpha} q}$ and $\mathbb{Z}_{q} \rtimes \mathbb{Z}_{p^{\alpha}}$ and these are all lattice-planar.

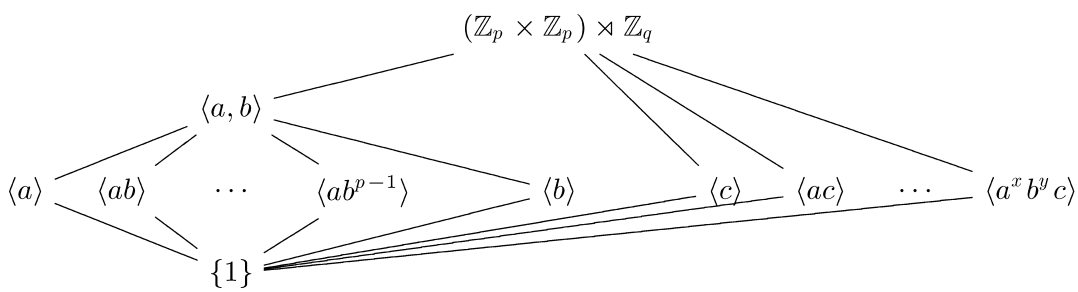

Fig. 16 Lattice of $\left(\mathbb{Z}_{p} \times \mathbb{Z}_{p}\right) \rtimes \mathbb{Z}_{q}$ 
Proof: Let $P$ denote a Sylow $p$-subgroup and $Q$ a Sylow $q$-subgroup.

We will induct on $\alpha$. Consider the case when $\alpha=3$. If $p>q$, then $n_{p}=1$ by Sylow's theorem and our group $G \cong P \rtimes \mathbb{Z}_{q}$. Since $P$ must be planar, we must have $P \cong \mathbb{Z}_{p^{3}}, \mathbb{Z}_{p^{2}} \times$ $\mathbb{Z}_{p}, M_{p^{3}}$, or $Q_{8}$. If $P \cong \mathbb{Z}_{p^{3}}$, we are done by Proposition 2.15 . If $P \cong \mathbb{Z}_{p^{2}} \times \mathbb{Z}_{p}$ or $M_{p^{3}}$, $a$ and $b$ are generators of $P$ ( $a$ of order $p^{2}, b$ of order $p$ ), and $c$ is a generator for $\mathbb{Z}_{q}$, then we have $c a c^{-1}=a^{i} b^{j}$ and hence $c a^{p} c^{-1}=a^{p i}$. Both $\mathbb{Z}_{p^{2}} \times \mathbb{Z}_{p}$ and $M_{p^{3}}$ have the characteristic subgroup $P^{\prime}=\left\langle a^{p}, b\right\rangle \cong \mathbb{Z}_{p} \times \mathbb{Z}_{p}$. In order for $P^{\prime} \rtimes Q$ to be planar, it must be isomorphic to Group 6 of order $p^{2} q$, but in this case the matrix appearing in the definition of Group 6 has the form $\left(\begin{array}{ll}i & 0 \\ k & \ell\end{array}\right)$. This matrix has eigenvalues in the field $\mathbb{F}_{p}$ and hence cannot have order dividing $p+1$ as required. Our last subcase is $P \cong Q_{8}$. It is well known that $\operatorname{Aut}\left(Q_{8}\right) \cong S_{4}$. If $q>3, q \nmid 24$ and our semi-direct product must be the direct product $Q_{8} \times \mathbb{Z}_{q}$ which has the nonplanar group $\mathbb{Z}_{2} \times \mathbb{Z}_{2} \times \mathbb{Z}_{q}$ as a quotient. If $q=3$, the only nontrivial semidirect product up to isomorphism is $Q_{8} \rtimes \mathbb{Z}_{3}=\langle a, b, c| a^{4}=b^{4}=c^{3}=1, a^{2}=$ $\left.b^{2}, b a b^{-1}=a^{-1}, c a c^{-1}=b, c b c^{-1}=a b\right\rangle$ [3, p. 159]. The figure below shows that this group is nonplanar.

Consider the case when $p<q$ and $(p, q) \neq(2,3)$. We cannot have $n_{q}=p$. If $n_{q}=p^{2}$, then $q \mid(p+1)(p-1)$ which implies that $q \mid(p+1)$ or $q \mid(p-1)$, but this is impossible since $q>p>2$. If $n_{q}=p^{3}$, then there are $p^{3}(q-1)$ elements of order $q$. But this only leaves $p^{3} q-p^{3}(q-1)=p^{3}$ elements and our Sylow $p$-subgroup must be normal, a case we have already considered. Therefore, the only remaining possibility is that we have a semi-direct product isomorphic to $\mathbb{Z}_{q} \rtimes P$, where $P \cong \mathbb{Z}_{p^{3}}, \mathbb{Z}_{p^{2}} \times \mathbb{Z}_{p}, M_{p^{3}}$, or $Q_{8}$. If $P \cong \mathbb{Z}_{p^{3}}$ the only nonabelian planar possibility is the one claimed (by Lemma 2.12). If $P \cong \mathbb{Z}_{p^{2}} \times \mathbb{Z}_{p}$ or $M_{p^{3}}$, each has a subgroup isomorphic to $\mathbb{Z}_{p} \times \mathbb{Z}_{p}$ which is nonplanar by Proposition 2.13. If $P \cong Q_{8}$ and the semi-direct product is a direct product, $n_{p}=1$ and we have already dealt with this case. According to [13, p. 257], up to isomorphism the only non-trivial semidirect product is $\left\langle a, b, c \mid a^{q}=b^{4}=c^{4}=1, b^{2}=c^{2}, b c b^{-1}=c^{-1}, b a=a b, c a c^{-1}=a^{-1}\right\rangle$. In this case $b^{2}$ is central and $G /\left\langle b^{2}\right\rangle$ is the nonplanar Group 2 of order $p^{2} q$.

Finally, when $(p, q)=(2,3)$ Burnside [3, p. 160] states that the only group of order 24 that is not a semi-direct product is $S_{4}$ which is nonplanar by Example 1.4. This completes the case when $\alpha=3$.

When $\alpha>3$ and $n_{p}=1$ our group is isomorphic to $P \rtimes \mathbb{Z}_{q}$ with $P$ planar. If $P \cong \mathbb{Z}_{p^{\alpha}}$, we are done by Proposition 2.14. If $P \cong \mathbb{Z}_{p^{\alpha-1}} \times \mathbb{Z}_{p}$ or $M_{p^{\alpha}}, P^{\prime}=\mathbb{Z}_{p^{\alpha-2}} \times \mathbb{Z}_{p}$ is characteristic in $P$ and $P^{\prime} \rtimes \mathbb{Z}_{q}$ is nonplanar by induction. We must also deal with the case when $p=2$ and $\alpha=4$ separately, since $P \cong Q_{16}$ or $Q D_{16}$ are also possible. But in both cases, $\left\langle a^{4}\right\rangle$ is characteristic and modding out by it gives $Q_{8} \rtimes \mathbb{Z}_{q}$ or $D_{8} \rtimes \mathbb{Z}_{q}$ which yields no planar groups.

When $\alpha>3$ and $n_{p}(G) \neq 1$, we know that there must be a normal subgroup $K \unlhd G$ of index $p$, since all groups of order $p^{\alpha} q^{\beta}$ are solvable [3, p. 323]. If $n_{p}(K) \neq 1$ we have at least three distinct Sylow $p$-subgroups of $K$, which must each lie in distinct Sylow $p$ -

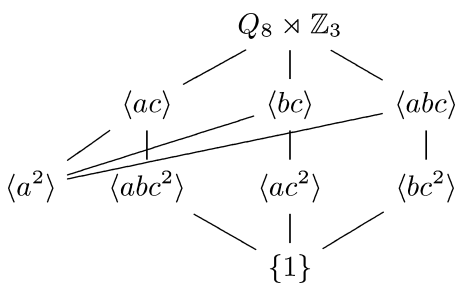

Fig. $17 K_{3,3}$ in the lattice of $Q_{8} \rtimes \mathbb{Z}_{3}$ 
subgroups $P_{1}, P_{2}, P_{3}$ of $G$. Applying Proposition $2.11, G$ is nonplanar. If $n_{p}(K)=1$, then $K=P^{\prime} \rtimes \mathbb{Z}_{q}$, which must be cyclic by induction. Since the intersection of any Sylow $q$ subgroup of $G$ with $K$ is a Sylow $q$-subgroup of $K$ [6, p. 147, ex. 34] and all of these $q$-subgroups have order $q$, this forces $n_{q}(G)=1$. Therefore $G \cong \mathbb{Z}_{q} \rtimes P$. If $P \cong \mathbb{Z}_{p^{\alpha}}$, we are done by Lemma 2.12. If $P \cong \mathbb{Z}_{p^{\alpha-1}} \times \mathbb{Z}_{p}$ or $M_{p^{\alpha}}$, then $\mathbb{Z}_{p} \times \mathbb{Z}_{p} \leq P$ and we are done by Proposition 2.13. It remains to deal with the case $p=2$ and $\alpha=4$, when $P \cong Q_{16}$ or $Q D_{16}$ are possibilities. The fact that $\mathbb{Z}_{2} \times \mathbb{Z}_{2} \leq Q D_{16}$ and $Q_{8} \leq Q_{16}$ reduces these to previously studied nonplanar cases.

\subsubsection{Groups of order $p^{2} q^{2}$}

Proposition 2.16. The only planar groups of order $p^{2} q^{2}$ are the cyclic groups.

Proof: As usual, $P$ and $Q$ will denote a Sylow $p$-subgroup and $q$-subgroups respectively. We may assume without loss of generality that $p>q$. By Sylow's Theorem, $n_{p}=1, q$, or $q^{2}$. We cannot have $n_{p}=q$ since $p>q$. If $n_{p}=q^{2}$, then $p \mid(q+1)(q-1)$ which implies that $p \mid(q+1)$. This is impossible unless $(p, q)=(3,2)$, a case we will address shortly. Except possibly for this case, we therefore have $G \cong P \rtimes Q$. If $G \cong \mathbb{Z}_{p^{2}} \rtimes \mathbb{Z}_{q^{2}}$, we are done by Proposition 2.14.

If $G \cong \mathbb{Z}_{p^{2}} \rtimes\left(\mathbb{Z}_{q} \times \mathbb{Z}_{q}\right)$, the subgroups $\mathbb{Z}_{p^{2}} \rtimes\left(\mathbb{Z}_{q} \times\{0\}\right)$ and $\mathbb{Z}_{p^{2}} \rtimes\left(\{0\} \times \mathbb{Z}_{q}\right)$ must be cyclic, but this forces $G \cong \mathbb{Z}_{p^{2}} \times\left(\mathbb{Z}_{q} \times \mathbb{Z}_{q}\right)$ which is nonplanar by Theorem 1.6.

If $G \cong\left(\mathbb{Z}_{p} \times \mathbb{Z}_{p}\right) \rtimes \mathbb{Z}_{q^{2}}$, then $G \cong\langle a, b, c| a^{p}=b^{p}=c^{q^{2}}=1, a b=b a, c a c^{-1}=$ $\left.a^{i} b^{j}, c b c^{-1}=a^{k} b^{\ell}\right\rangle$, where $M=\left(\begin{array}{ll}i & j \\ k & \ell\end{array}\right)$ has order dividing $q^{2}$ in $G L_{2}(p)$. The subgroup $\left\langle a, b, c^{q}\right\rangle$ must be planar and hence must be isomorphic to Group 6 of order $p^{2} q$. The matrix associated to that group is $M^{q}$ which must have order $q$, hence $M$ has order $q^{2}$. Any element of the form $a^{i} b^{j} c^{k}$ has order $q^{2}$ when $q \nmid k$. Figure 18 gives a nonplanar sublattice.

If $G \cong\left(\mathbb{Z}_{p} \times \mathbb{Z}_{p}\right) \rtimes\left(\mathbb{Z}_{q} \times \mathbb{Z}_{q}\right)$, it contains the nonplanar group $\left(\mathbb{Z}_{p} \times \mathbb{Z}_{p}\right) \times \mathbb{Z}_{q}$ as a subgroup, regardless of the automorphism used to define the semidirect product [7].

Finally, we must deal with the case when $(p, q)=(3,2)$ and $n_{p} \neq 1$. A standard argument, e.g. [7, p. 176], shows that in that case there is a surjective homomorphism from $G$ to $A_{4}$. Let $H$ be the inverse image of $\mathbb{Z}_{2} \times \mathbb{Z}_{2} \leq A_{4}$. Now $H$ is planar, it has order 12 and a normal subgroup $K$ of order 3 such that $H / K \cong \mathbb{Z}_{2} \times \mathbb{Z}_{2}$. The only planar groups of order 12 are $\mathbb{Z}_{12}, A_{4}$, and $T=\mathbb{Z}_{3} \rtimes \mathbb{Z}_{4}$. But $A_{4}$ has no normal subgroups of order 3 and the quotient of $A_{4}$ or $T$ by its unique normal subgroup of order 3 is $\mathbb{Z}_{4}$, not $\mathbb{Z}_{2} \times \mathbb{Z}_{2}$.

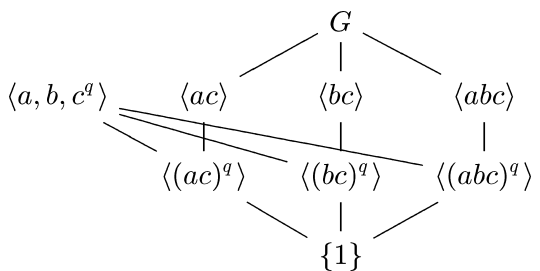

Fig. $18 K_{3,3}$ in a group of order $p^{2} q^{2}$ 


\subsubsection{Groups of order $p^{\alpha} q^{\beta}, \alpha, \beta \geq 2$}

Proposition 2.17. The only planar groups of order $p^{\alpha} q^{\beta}, \alpha, \beta \geq 2$, are the cyclic groups.

Proof: Let $G$ denote our group of order $p^{\alpha} q^{\beta}$. We will induct on $\alpha+\beta$ beginning with $\alpha+\beta=4$, where the result holds by Proposition 2.16. Now suppose $\alpha+\beta>4$. Since $G$ is solvable, it must contain a normal subgroup $H$ of prime index, without loss of generality, say $q$. As usual, $P$ will denote a Sylow $p$-subgroup and $Q$ a Sylow $q$-subgroup.

If $\beta>2$, then $H$ must be cyclic by induction. Since the intersection of a Sylow $p$-subgroup of $G$ with $H$ yields a Sylow $p$-subgroup of $H$, the Sylow $p$-subgroups of $G$ and of $H$ have order $p^{\alpha}$, and $n_{p}(H)=1$, we must have $n_{p}(G)=1$. Therefore $G \cong P \rtimes Q$ with $P$ cyclic. There is a characteristic subgroup $P^{\prime} \leq P$ of index $p$ and $K=P^{\prime} \rtimes Q \leq P \rtimes Q$, so $K$ is planar. If $\alpha>2, K$ is cyclic by induction and if $\alpha=2, K$ is cyclic or isomorphic to $\mathbb{Z}_{p} \rtimes \mathbb{Z}_{q^{\beta}}$ by Proposition 2.15. In either case, $Q$ must be cyclic, and hence $G \cong P \rtimes Q$ must be cyclic by Proposition 2.14 .

If $\beta=2$, then $H$ is cyclic, and we may proceed as above, or $H \cong \mathbb{Z}_{q} \rtimes \mathbb{Z}_{p^{\alpha}}$ by Proposition 2.14. In this case, $\mathbb{Z}_{q}$ is characteristic in $H$, hence normal in $G$. We have two subcases to consider. If $n_{q}(G) \neq 1$, let $L=G / \mathbb{Z}_{q}$. We have $|L|=p^{\alpha} q$ and $n_{q}(L) \neq 1$ since $\mathbb{Z}_{q}$ lies in all of the Sylow $q$-subgroups of $G$, but no planar groups of this order have $n_{q} \neq 1$. If $n_{q}(G)=1$, then $G=Q \rtimes P$. If $Q$ is cyclic, we use the arguments in the previous paragraph. If $Q$ is not cyclic, we use the fact that $P$ contains a subgroup $P^{\prime}$ of order $p^{2}$ and invoke Proposition 2.16 to show that $Q \rtimes P^{\prime}$ is not planar.

\subsection{Groups of order $p^{\alpha} q^{\beta} r^{\gamma}$}

Proposition 2.18. The only solvable planar groups of order $p^{\alpha} q^{\beta} r^{\gamma}$ are the cyclic ones (those in which two of the exponents are 1).

Proof: Let $G$ be our group. If $n_{p}=n_{q}=n_{r}=1, G$ is the direct product of its Sylow subgroups. Since $P \times Q, P \times R$ and $Q \times R$ must be planar, by our work on groups of order $p^{\alpha} q^{\beta}$, the Sylow subgroups must be cyclic. Therefore, $G$ is cyclic. We know this group is only planar when two of the exponents are 1 by Theorem 1.6.

Without loss of generality, suppose $n_{p} \neq 1$. Let $P, Q$ and $R$ form a Sylow basis for $G$, and let $P^{\prime}$ be a Sylow subgroup distinct from $P$. Since $P$ and $P^{\prime}$ are distinct and $P Q \cap P R=P$,

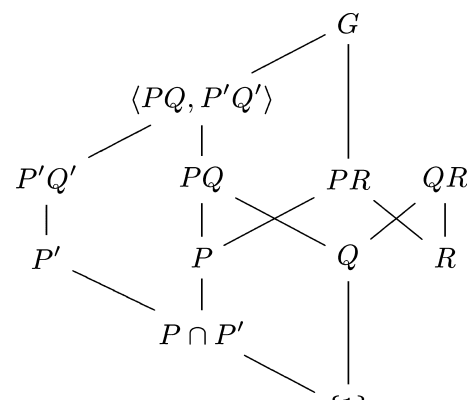

$\{1\}$

Fig. $19 K_{3,3}$ for one case in Proposition 2.18 
we cannot have $P^{\prime} \leq P Q \cap P R$. This implies that there cannot be exactly one Hall subgroup of order $p^{\alpha} q^{\beta}$ and exactly one of order $p^{\alpha} r^{\gamma}$. Assume that $P Q \neq P^{\prime} Q^{\prime}$ are Hall subgroups of order $p^{\alpha} q^{\beta}$. The edges from $P$ to $P Q$ and from $P^{\prime}$ to $P^{\prime} Q^{\prime}$ may or may not cross. If they do not, Figure 19 shows a $K_{3,3}$ in the subgroup graph. Note that $\left\langle P Q, P^{\prime} Q^{\prime}\right\rangle$ might be $G$ and $P \cap P^{\prime}$ might be trivial, but this does not affect the nonplanarity.

If the edges do cross, we repeat the the construction above with the roles of $Q$ and $R$ reversed. This is only a problem if the edges from $P$ to $P R$ and from $P^{\prime}$ to $P^{\prime} R^{\prime}$ also cross. But in that case, $P^{\prime} \leq P Q \cap P R$, which we have seen is impossible.

This completes the classification of planar finite solvable groups.

\section{Nonsolvable groups}

Recall that a minimal simple group is a nonabelian simple group all of whose proper subgroups are solvable.

Since any nonsolvable group has a simple group as a subquotient and every simple group has a minimal simple group as a subquotient, if we can show that the minimal simple groups are nonplanar, we will have shown that the nonsolvable groups are nonplanar. The classification of minimal simple groups, which preceded the full classification of simple groups historically, will be given below.

Definition 3.1. Recall that $S L_{m}(n)$ is the group of $m \times m$ matrices having determinant 1 whose entries lie in a field with $n$ elements and that $L_{m}(n)=S L_{m}(n) / H$ where $H=$ $\left\{k I \mid k^{m}=1\right\}$.

Theorem 3.2. A finite group is a minimal simple group if and only if it is isomorphic to one of the following:

1. $L_{2}\left(2^{p}\right)$ ( $p$ any prime)

2. $L_{2}\left(3^{p}\right)$ ( $p$ an odd prime)

3. $L_{3}(3)$

4. $L_{2}(p)$ where $p^{2} \equiv-1 \bmod 5$ and $p>3$,

5. $\mathrm{Sz}\left(2^{q}\right)$ with $q \geq 3$ and odd.

Proof: [12, p. 388]. All we need to know about $\mathrm{Sz}\left(2^{q}\right)$, known as Suzuki groups, will be quoted below.

Lemma 3.3. The dihedral groups $D_{4 n}$ are nonplanar for $n>2$.

Proof: If $p$ is a prime that divides $n,\left\langle a^{2 n / p}, b\right\rangle \cong D_{4 p}$. This was shown to be nonplanar in Proposition 2.16 where it was Group 4 of order $4 p$.

Theorem 3.4. There are no nonsolvable planar groups.

Proof: As noted above, it will suffice to show that the minimal simple groups are nonplanar. We will denote the image of a matrix $A$ in $L_{m}(n)$ by $\bar{A}$.

$\boldsymbol{L}_{\mathbf{2}}\left(\boldsymbol{q}^{p}\right)$ : We take care of the first two families on our list. For $p=2$, the only candidate is $q^{p}=4$. Note first that $L_{2}(4) \cong A_{5}$ [1] which is nonplanar by Example 1.4. 
For $p>2$, we have that $L_{2}\left(q^{p}\right)$ contains a nonplanar subgroup isomorphic to $\left(\mathbb{Z}_{q}\right)^{p}$, namely the subgroup of matrices of the form $\left(\begin{array}{ll}1 & a \\ 0 & 1\end{array}\right)$ with $a \in \mathbb{F}_{q^{p}}$.

$\boldsymbol{L}_{\mathbf{3}}(3)$ : In $S L_{3}(3)$ the only matrix in the subgroup $H$ is the identity matrix, so $L_{3}(3) \cong$ $\mathrm{SL}_{3}(3)$. Consider the subgroup consisting of matrices of the form

$$
\left(\begin{array}{lll}
1 & a & b \\
0 & 1 & c \\
0 & 0 & 1
\end{array}\right)
$$

with $a, b, c \in \mathbb{F}_{3}$. This subgroup is isomorphic to the group $\left(\mathbb{Z}_{p} \times \mathbb{Z}_{p}\right) \rtimes \mathbb{Z}_{p}$ with $p=3$ which is nonplanar by Lemma 2.5 (Figure 6).

$\boldsymbol{L}_{\mathbf{2}}(\boldsymbol{p})$ : Note that here $H=\{ \pm I\}$. We must deal with two cases.

Case 1: $p \equiv 1 \bmod 4$ : We will show that $L_{2}(p)$ contains a subgroup isomorphic to $D_{p-1}$. Let $x \in \mathbb{Z}_{p}$ be a primitive root and $y \in \mathbb{Z}_{p}$ an element such that $y^{2} \equiv-1 \bmod p$ (which exists since $p \equiv 1 \bmod 4)$. Let $a \equiv 2^{-1}\left(x+x^{-1}\right) \bmod p$ and $b \equiv(2 y)^{-1}\left(x-x^{-1}\right)$ mod $p$, $R=\left(\begin{array}{cc}a & b \\ -b & a\end{array}\right)$, and $S=\left(\begin{array}{cc}y & 0 \\ 0 & -y\end{array}\right)$. It is straightforward to verify that $R, S \in S L_{2}(p)$ and that $\langle\bar{R}, \bar{S}\rangle \stackrel{-b}{=}\left\langle r, s \mid r^{(p-1) / 2}=s^{2}=1, s r s^{-1}=r^{-1}\right\rangle \cong D_{p-1}$, which is nonplanar by Lemma 3.3 when $p>5$. When $p=5, L_{2}(5) \cong A_{5}$ [1] which is nonplanar by Example 1.4.

Case 2: $p \equiv 3 \bmod 4$ : We will show that $L_{2}(p)$ contains a subgroup isomorphic to $D_{p+1}$. Since $p \equiv 3 \bmod 4, x^{2}+1$ is irreducible over $\mathbb{F}_{p}$ and we can identify the field $\mathbb{F}_{p^{2}}$ with $\mathbb{F}_{p}[x] /\left(x^{2}+1\right)$. The multiplicative group of this field is cyclic of order $p^{2}-1$, so there must be an element, $y=a+b x$, of order $p+1$ in this group. Let $R=\left(\begin{array}{cc}a & b \\ -b & a\end{array}\right)$. There are elements $s, t \in \mathbb{F}_{p}$ such that $s^{2}+t^{2} \equiv-1 \bmod p\left[4, \mathrm{p}\right.$. 1]. Let $S=\left(\begin{array}{cc}s & t \\ t-s\end{array}\right)$. It may be verified that $R, S \in S L_{2}(p)$ and that $\langle\bar{R}, \bar{S}\rangle=\left\langle r, s \mid r^{(p+1) / 2}=s^{2}=1, s r s^{-1}=r^{-1}\right\rangle \cong D_{p+1}$, which is nonplanar when $p>7$. For $p=7, S_{4}$ is a maximal subgroup of $L_{2}(7)$ [1] so it is nonplanar by Example 1.4.

$\mathbf{S z}\left(2^{q}\right)$ : The Suzuki group, $\operatorname{Sz}\left(2^{q}\right)$, contains a subgroup isomorphic to $\left(\mathbb{Z}_{2}\right)^{q}$ [5, p. 466]. Since $q \geq 3$ this subgroup is nonplanar by Theorem 1.6.

\section{Conclusion}

Putting the results of the previous sections together we obtain our main theorem.

In [11], Starr and Turner also classify the infinite abelian planar and lattice-planar groups. We know of no examples of infinite nonabelian planar groups. The question of their existence or non-existence and the study of other graph-theoretical properties of the subgroup graph will be the subject of future work.

Added in proof: Ol'shanskii's construction of "Tarski monster" groups, infinite groups all of whose proper subgroups have fixed prime order, yields examples of infinite nonabelian planar groups.

Acknowledgement The authors would like to thank the referees, whose comments greatly improved the exposition of this paper. 


\section{References}

1. Atlas of Finite Group Representations, http://web. mat.bham.ac .uk/atlas/v2 .0/.

2. J.P. Bohanon, The Planarity of Hasses Lattices of Finite Groups, Master's Thesis, Southwest Missouri State University, 2004.

3. W. Burnside, Theory of Groups of Finite Order, Dover Publications, Cambridge, 1955.

4. D.A. Cox, Primes of the Form $x^{2}+n y^{2}$, John Wiley, New York, 1989.

5. D. Gorenstein, Finite Groups, Harper and Row, New York, 1968.

6. D.S. Dummit and R.M. Foote, Abstract Algebra, 3rd ed., John Wiley and Sons, 2004.

7. H.-L. Lin, "On groups of order $p^{2} q, p^{2} q^{2}$," Tamkang J. Math. 5 (1974), 167-190.

8. C.R. Platt, "Planar lattices and planar graphs," J. Combinatorial Theory Ser. B 21 (1976), 30-39.

9. F.S. Roberts, Applied Combinatorics, Prentice-Hall, Englewood Cliffs, NJ, 1984.

10. W.R. Scott, Group Theory, Dover, New York, NY, 1964.

11. C.L. Starr and G. E. Turner III, "Planar groups," J. Algebraic Combin. 19 (2004), 283-295.

12. J.G. Thompson, "Nonsolvable finite groups All of whose local subgroups are solvable," Bull. Amer. Math. Soc. 74 (1968), 383-437.

13. A.E. Western, "Groups of order $p^{3} q$," Proc. London Math. Soc. 30 (1899), 209-263.

14. J.B. Wilson, Planar Groups, http://darkwing.uoregon.edu/ jwilson7/math/ planargroups.html. 